\title{
Article
}

\section{Fully-Autonomous Operation of an AC Micro-grid with Inherent Seamless Switching}

\author{
Baoquan Liu ${ }^{1}$, Yan Liu ${ }^{1}$, Yixin Zhu ${ }^{2}$ and Yong Shi * \\ 1 School of Electrical and Information Engineering, Shaanxi University of Science and Technology, Xi'an, \\ Shaanxi, 710021, China; liubq@sust.edu.cn; liuyan@sust.edu.cn and shiyong@sust.edu.cn \\ 2 College of Internet of Things, Jiangnan University, Wuxi, Jiangsu Province, 214122, China; \\ zhuyixin1987@163.com \\ * Correspondence: shiyong@sust.edu.cn and liubq@sust.edu.cn
}

\begin{abstract}
This paper proposes a novel micro-grid structure, which can operate fully-autonomously with inherent seamless switching. It can operate independently in both grid-connected and islanded mode as a self-governed entity without relying on the utility grid. An AC/DC/AC converter is employed as the interface between the micro-grid and the utility grid, which enables the two entities to have different voltages in grid-connected mode. Seamless switching between operation modes can be achieved naturally. The micro-grid is regulated to exchange predefined amount of power with the utility grid in grid-connected mode. This will benefit the power dispatching algorithm of the power system. The predefined power is estimated based on power forecasting of local renewable generations and loads with consideration of the Sate of Charge (SOC) of the battery, and is updated and broadcasted every certain period. A small scale AC micro-grid with a rotating generator, battery storage and solar arrays etc. is built for investigation. Matlab/Simulink results are provided to validate the robustness and flexibility of proposed micro-grid and its operation strategy.
\end{abstract}

Keywords: Fully-autonomous; AC micro-grid; AC/DC/AC converter; Seamless switching

\section{Introduction}

Distributed generations (DGs), such as solar arrays and wind turbines has increased significantly in the past decades. Then micro-grid has eventually become a promising solution for distributed energy harvesting and utilizations, especially in rural and remote areas, where the utility grid cannot reach [1]-[3]. Micro-grid is normally a low voltage network, which incorporates DGs, local loads, energy storage and other auxiliary infrastructures, aiming to power a certain area (e.g. an offshore island, a residential community or a rural pasture). It may have different structures and functionalities according to the application scenarios [4]-[6].

Regarding to the operation strategy, during islanded mode, a micro-grid operates autonomously. Whereas in grid-connected mode, the system always rely on the utility grid in BUS voltage supporting and power balancing [7]-[10]. In [7], during grid-connected mode, the AC BUS is dominated by the utility grid and all DGs work as grid-following units with PQ controllers. The power flow is balanced automatically by the utility grid. When it turns to islanded mode, the BUS voltage source transfers to one of its storage units with droop controllers, and the system operates self-sufficiently. Similar operation strategy is also applied in [8], and to achieve seamless switching, a Control Area Network based grid synchronization technique is proposed. This kind of operation methodologies have several disadvantages:

(1) Seamless switching problem

The AC BUS voltage is dominated by the utility grid during grid-connected mode and hands over to its subunits when it comes to islanded mode. This islanding action may introduce system uncertainties even lead to instability problems.

Before reconnecting, both of the two entities are voltage sources. Grid synchronization and surge current mitigation become great concerns, calling for advanced technologies. 
(2) Multiple system operation strategies

The islanded mode is autonomous, whereas the grid-connected mode is actually nonautonomous. Then multiple system operation strategies may be required and change along with the operation mode switching.

(3) Power exchange fluctuation

During grid-connected mode, system operation relies on the utility grid in power balancing. The power exchange between them is fluctuating due to the intermittency of the renewable sources and local loads. This will complicate the power dispatching algorithm of the power system and increase instability risks, especially when deeply penetrated.

Recent works have developed plenty of approaches to improve the seamless switching process typically in droop-based AC micro-grids [11]-[13]. With advanced grid-synchronization and control technologies, transition dynamics in mode switching can be mitigated effectively. Furthermore, only a single kind of operation strategy is required, which qualifies both operation modes. In [12], a voltage-based droop control algorithm is proposed, where a specific DG unit with sufficient capacity is regulated to achieve grid-synchronization for the reconnecting action. Reference [13] has proposed a variable structure based internal model voltage controller and an active damping based droop controller for seamless switching and power sharing. These works contribute greatly to the operation of micro-grids, especially in mode transitions, but the dependence on utility grid in power balancing during grid-connected mode remains. The power exchange is always varying due to the intermittency of renewable sources and local loads, which hinders the large-scale application of micro-grids within the utility grid.

To integrate large quantities of micro-grids that involving renewable generations into the utility grid and also improve the seamless switching between operation modes, a fully-autonomous $\mathrm{AC}$ micro-grid framework and its operation strategy is proposed in this work. An AC/DC/AC back-toback power electronic converter with two basic three-phase voltage source converters (VSC) is constructed to interface the micro-grid to the utility grid. With the DC link in-between, the two entities can possess different voltage amplitudes, frequencies and phase angles.

In the operation strategy, one VSC of the AC/DC/AC converter works as a rectifier to maintain the DC link voltage stable and the other VSC is regulated as an inverter. Seamless switching is achieved naturally with a single kind of operation strategy. Furthermore, all the volatile power components from renewable sources and local loads are constrained and suppressed within the micro-grid circumstances, and the micro-gird entity is controlled to exchange predefined amount power with the utility grid during grid-connected mode and zero during islanded mode. The power exchange amount is estimated based on power forecasting of local renewable generations and loads with consideration of the SOC of the battery, and is updated and broadcasted every certain period. In this case, the micro-grid behaves as a predictable node for the power system, benefiting the power dispatching algorithm and further high penetration depth applications.

A similar concept is concisely demonstrated with a DC micro-grid in author's another work, but AC fully-autonomous micro-grids and the operation strategy is not involved [14]. Furthermore, the estimation of the power exchange amount based on power forecasting is not investigated. The rest of this work is organized as follows. Section II describes the AC micro-grid layout and the operation strategy including the control of the AC/DC/AC converter and the battery storage. Section III illustrates the power exchange estimation based on power forecasting and section IV presents the simulation results in typical scenarios on Matlab/Simulink platform. Finally, section V draws the conclusions

\section{System Layout and Operation Strategy}

\subsection{System Layout}

In this work, typical elements are chosen to build an AC micro-grid as the object for investigation. The configuration is schematically displayed in Fig.1. It consists of the following units, which are commonly the most preferred choices in micro-grid constructions. 
- Diesel genset, to represent the traditional rotating generations, which is still the de-facto power supply for emergency and remote area utilizations [15];

- Battery, which is the most used storage device for power smoothing and load shifting in microgrids;

- $\quad$ Solar array, a promising renewable energy DG, who provides intermittent power according to weather conditions;

- $\quad$ Residential loads, critical RL load and noncritical converter load are included;

- $\quad$ Point of Common Coupling (PCC), which is the only joint of between the two entities;

- Transformer, to achieve electric isolation between the utility grid and the micro-grid.

Compared with conventional AC micro-grids, the most prominent and unique feature of the proposed framework in system structure is that an $\mathrm{AC} / \mathrm{DC} / \mathrm{AC}$ converter is utilized as the interface unit. The AC/DC/AC converter has a DC link in-between, which enables the two entities to operate with different voltage amplitudes, frequencies and phase angles. Control algorithm of this AC/DC/AC converter will be detailed later. The power flow of each unit in this micro-grid are defined as $P_{d e}, P_{b a t}, P_{P V}, P_{l}$ and $P_{e x}$, and the line impedance are defined as $Z_{1} \sim Z_{4}$.

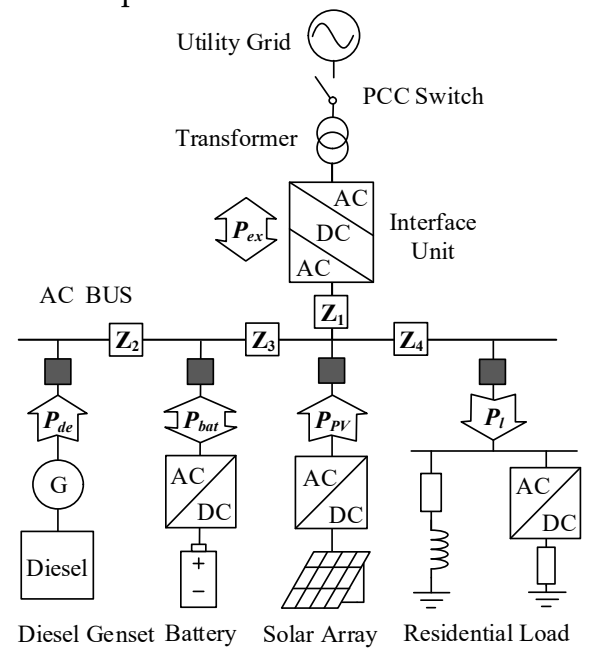

Figure 1. System configuration and layout of the target AC micro-grid.

In operation design, the AC BUS of the micro-grid is dominated by its subunits (diesel genset in this work) all along in both grid-connected and islanded mode. Then the micro-grid can operate autonomously in both operation modes without relying on the utility grid in AC BUS supporting, which indicates a "fully-autonomous" framework.

Normally, grid-forming of a micro-grid can adopt droop-based control or V/f control with or without communications [16]-[18]. Reference [16] has proposed a secondary control loop to restore the voltage amplitude and frequency in a droop-based AC micro-grid. In [17], centralized control is designed to manage the power flow in a master/slave type micro-grid. In this work, the diesel genset is assigned to perform as the master unit for AC BUS supporting. The solar array unit works under the control of a Maximum Power Point Tracking (MPPT) algorithm with curtailment design in extreme conditions. All the volatile power fluctuations from solar and residential loads are supposed to be constrained and suppressed within the micro-grid circumstances. The battery storage unit will take the responsibility in power smoothing.

The AC/DC/AC converter is regulated to absorb/deliver predefined amount of power from/to the utility grid in every certain period. The amount of this power exchange is estimated based on power forecasting of renewable generations and local loads with consideration of the SOC of the battery storage, which are illustrated in next section. 


\subsection{Control of the Diesel Genset}

The diesel genset works as the grid-forming unit of the micro-grid to support the main AC BUS. The diesel engine is equipped with a speed governor (controller and actuator in Fig.2), which ensures frequency regulation of the output voltages. Furthermore, the static exciter of the generator ensures the constant amplitude of the output voltages. The control scheme is schematically displayed in Fig.2, where two feedback control loops ( $\omega$ in pu and $E$ in pu) are included.

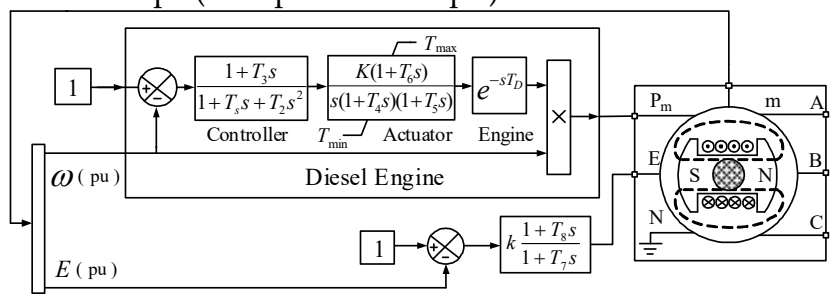

Figure.2. Schematic and control of the diesel generator set.

\subsection{Control of the AC/DC/AC Converter}

The topology of the AC/DC/AC converter is displayed in Fig.3. Two basic three-phase VSCs are connected back-to-back in series with a DC link in-between. Through the AC/DC/AC converter and the PCC switch as well as an isolating transformer (not showed in Fig.3), the micro-grid is connected to the utility grid indirectly. Each VSC can work bi-directionally, and in this AC/DC/AC converter, VSC_\#1 works as a rectifier to maintain the DC link voltage and VSC_\#2 works as an inverter to transmit power from the DC link to the utility grid.

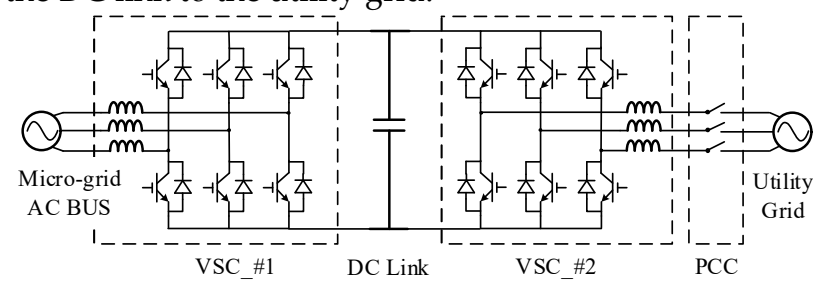

Figure.3. Topology of the AC/DC/AC converter.

The control scheme of a general VSC is presented in Fig.4. The control algorithm is carried out under synchronous rotating reference frame $(d-q-0)$, where current ( $i_{d}$ and $\left.i_{q}\right)$ and voltage control (vDC) loops are designed. The phase angle $\varphi$ of AC side voltages $\left(e_{a}, e_{b}, e_{c}\right)$ is firstly estimated through a phased-lock-loop (PLL) algorithm. As displayed in Fig.5, $\left(e_{a}, e_{b}, e_{c}\right)$ are transformed to $e_{d}$ and $e_{q}$ under $d-q-0$ frame with a transformation matrix $T_{a b c / d q 0}$ in (1). $\omega_{f}$ is the nominal frequency of the AC voltages, which is a constant, and $\omega_{a}$ is the estimated frequency. By designing a feedback control loop, $e_{q}$ is regulated to zero and phase angle $\varphi$ is finally derived.

The estimated phase angle $\varphi$ of $\left(e_{a}, e_{b}, e_{c}\right)$ is used for the rotating transformation of inductor currents $\left(i_{a}, i_{b}, i_{c}\right)$, and $\left(i_{d}, i_{q}\right)$ is then calculated. $i_{d}$ is recognized as the active current, which aligns with the voltage vector and id is the reactive current, which is orthogonal to the voltage vector. Then current control loops of both channels are designed with PI controllers ( $\mathrm{PI}_{2}$ and $\left.\mathrm{PI}_{3}\right)$. Furthermore, to maintain the DC link voltage, a DC voltage control loop $\left(v_{D C}\right)$ is designed outer the active current loop $\left(i_{d}\right)$ accordingly. 


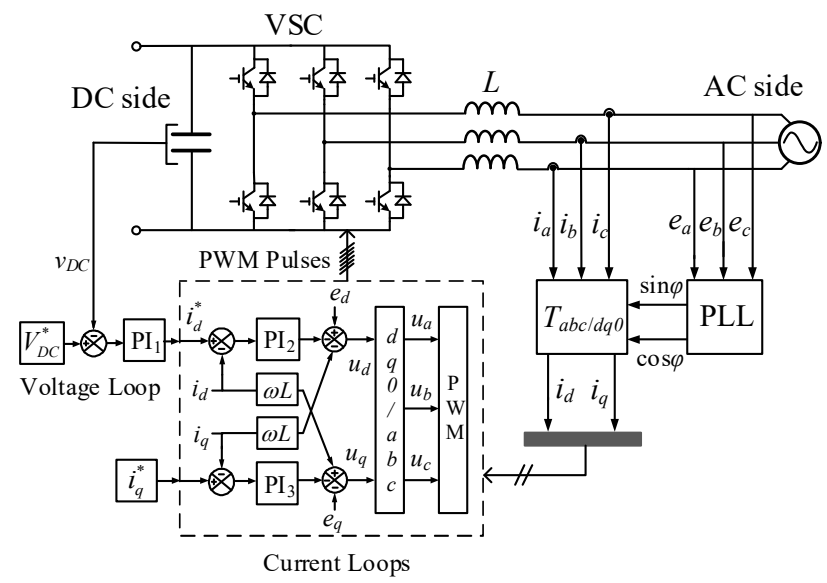

Figure.4. Control scheme of a VSC under d-q-0 frame.

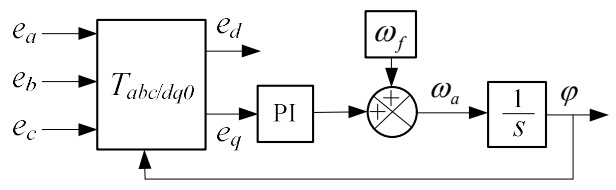

Figure. 5. Phase locked loop (PLL) in a three-phase VSC.

$$
T_{a b c l d q 0}=\frac{2}{3}\left[\begin{array}{ccc}
\cos (\varphi) & \cos \left(\varphi-\frac{2 \pi}{3}\right) & \cos \left(\varphi+\frac{2 \pi}{3}\right) \\
-\sin (\varphi) & -\sin \left(\varphi-\frac{2 \pi}{3}\right) & -\sin \left(\varphi+\frac{2 \pi}{3}\right) \\
\frac{1}{2} & \frac{1}{2} & \frac{1}{2}
\end{array}\right]
$$

Control of VSC_\#1 applies the same scheme in Fig.4. The micro-grid AC BUS is dominated by the diesel genset all along, then the voltages and currents of the micro-grid are metered for transformation. VSC_\#1 works as a rectifier and the DC link voltage is maintained constant VDC in both grid-connected and islanded mode through the voltage control loop.

Control of VSC_\#2 only applies the current control loops in Fig.4 and it works as an inverter. During islanded mode, the PCC switch is off, active and reactive current references are all set to zeros, $\left[i_{\mathrm{d} \neq 22}^{*} i_{\mathrm{q}^{\sharp 2}}^{*}\right]=[0,0]$. When it comes to grid-connected mode, the PCC switch is on and the AC side of VSC_\#2 is directly connected to the utility gird. VSC_\#2 is regulated to deliver/absorb predefined amount of power in every certain period. In implementation, active and reactive power $(P$ and $Q)$ control are translated into current ( $i_{d}$ and $i_{q}$ ) control through (2) and (3).

$$
\begin{aligned}
& i_{d \# 2}^{*}=\frac{2}{3} \frac{P_{e x}^{*}}{e_{d}} \\
& i_{q \# 2}^{*}=-\frac{2}{3} \frac{Q_{e x}^{*}}{e_{d}}
\end{aligned}
$$

where $P_{e x}^{*}$ and $Q_{e x}^{*}$ are the predefined active and reactive power set-points for exchanging with the utility grid.

As described above, the AC BUS of the micro-grid is dominated by its subunits (diesel genset) in both grid-connected and islanded mode. And the AC micro-grid operates independently as a selfgovern system all along. In mode switching, control of the AC/DC/AC converter does not require grid synchronization. The only difference between the two operation modes is the current loop reference set-points of VSC_\#2, predefined values in grid-connected mode and zero in islanded mode. Other units of the micro-grid does not necessarily change their present steps. Seamless switching is achieved naturally and only a single kind of operation strategy is required. Fully-autonomous operation of a micro-grid is achieved consequently in this proposed framework. 


\subsection{Control of the Battery Unit}

The battery storage is regulated by another VSC and it is supposed to undertake all the volatile power components. The power flows within the micro-grid is firstly analyzed. Regarding to the diesel genset, in low load-rate scenarios (below 30\% of the rated capacity), it generates excessive exhaust gases due to the incomplete combustion of fuels, which also leads to premature aging of the engine due to carbon deposit [15],[19]. To avoid low load-rate running, we prescribe a minimum power output limit to about $30 \%$ of its rated capacity, defined as $P_{D E}$.

The residential load includes a critical $R L$ part, which equals to the equipped $P_{D E}$. And the total residential load is defined as $P_{l}$ as depicted in Fig.1. The fluctuating power of the solar array is defined as $P_{P V}$. The predefined amount of power for exchanging with the utility grid is $P_{e x}$, which is a constant in a certain period. And also define $P_{e x}$ that flows from the micro-grid to the utility grid as positive. Then the total net power of the micro-grid $P_{\text {net }}$ is expressed as (4).

$$
P_{n e t}=P_{D E}+P_{P V}-P_{l}-P_{e x}
$$

Then $P_{\text {net }}$ is assigned to the battery units for compensation with consideration of the battery restrictive rules in (5).

$$
\left\{\begin{array}{c}
P_{D I S}<P_{b a t}<P_{C H A} \\
V_{L O W}<v_{b a t}<V_{U P} \\
S O C_{L O W}<S O C<S O C_{U P}
\end{array}\right.
$$

where $P$ bat is the power flowing into/out of the battery, vbat is the battery terminal voltage and SOC is its state of charge. Upper and lower limits are designed for the three variables, which are $\left[P_{D I S}, P_{C H A}\right]$, [VLOW, VuP ] and [SOCLOW, SOCuP].

When $P_{\text {net }} \geq 0, P_{\text {net }}$ should be totally charged into the battery without exceeding Р РнA. Moreover, if battery terminal voltage vbat reaches $V u p$, then $V u p$ should be maintained and $P_{b a t}$ begins to decrease. In extreme conditions, such as intense sun radiation but low load-rate, curtailment of the solar array will be activated for protection. When $P_{n e}<0$, the battery is discharged to compensate the power deficit until to its maximum capability $P_{D I S}$. If the load is till increasing, then the power gap will be filled up by the diesel genset automatically. This power sharing process is concisely presented in Fig.6, where two profiles $P_{b a t}$ and $P_{n e t}$ are included. Segment A and B are the curtailed power in extreme conditions. $C$ and $D$ is the power sharing of battery according to the first two rules in (5). Particularly, in $\left[t_{1}, t_{2}\right]$, the battery terminal voltage has reached Vup and Pbat begins to decrease. E and F is the load increment that is afforded by the diesel genset.

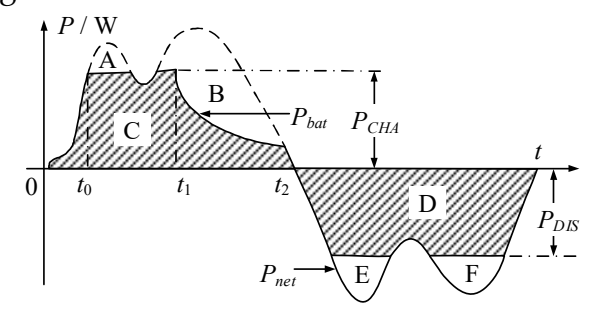

Figure.6. Power sharing of the battery storage unit.

The above power sharing process is realized by controlling the battery VSC as a rectifier. Some modifications are made on basis of the control block in Fig.4, getting a new scheme as displayed in Fig.7. In the voltage loop, terminal voltage of the battery vbat is metered as the feedback. Constant upper and lower limits of $\mathrm{PI}_{4}, P_{C H A}$ and $P_{D I S}$, has determined the maximum charging and discharging scope of the battery. Its output is defined as $P_{\text {bat }}^{\prime}$ and passes through a saturation block, who has a

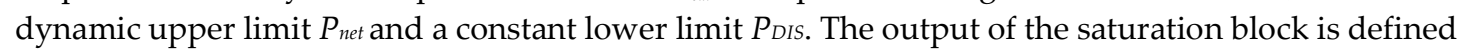
as $P_{b a t}^{*}$ and it will be the active power set-point for the battery VSC. 


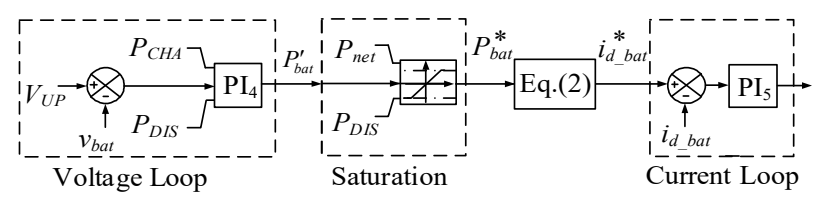

Figure. 7. Control of the battery VSC.

Analysis of this modified scheme is provided. If $P_{n e t} \geq 0$ and $v_{b a t}<V_{u p}, \mathrm{PI}_{4}$ outputs its maximum value $P_{\text {Сна. }}$. Through the saturation block, $P_{b a t}^{*}$ is derived by (6).

$$
P_{\text {bat }}^{*}=\min \left(P_{C H A}, P_{n e t}\right)
$$

When the battery terminal voltage vbat reaches $V u p$, the output of $\mathrm{PI}_{4}$ will be a positive value between 0 and $P_{\text {CHA }}$ to maintain $v_{b a t}$ at $V u p$. $P_{b a t}^{*}$ is derived by (7).

$$
P_{b a t}^{*}=\min \left(P_{b a t}^{\prime}, P_{n e t}\right)
$$

According to (6) and (7), when $P_{\text {net }} \geq 0, P_{b a t}^{*}$ equals to the smaller value between $P_{b a t}^{\prime}$ and $P_{\text {net }}$ without exceeding $P_{C H A}$ as well as the upper voltage limit VuP.

If $P_{n e t}<0$, the battery is discharged and $V_{C H A}>v b a t$ is always satisfied. Then the output of $\mathrm{PI}_{4}$ will be the maximum value Р СнA. Though the dynamic saturation block, $P_{b a t}^{*}$ can be derived by (8). $P_{b a t}^{*}$ equals to the larger value between $P_{D I S}$ and $P_{n e t}$, which indicates that the battery is discharge to compensate $P_{\text {net }}$ without exceeding $P_{\text {DIS. }}$

$$
P_{\text {bat }}^{*}=\max \left(P_{D I S}, P_{\text {net }}\right)
$$

Therefore, with the voltage loop and saturation block in Fig.7 as well as the above three equations, power sharing of the battery can be achieved with consideration of the first two restrictive rules in (5). Practically, in the control process of the battery VSC, similar to the VSC_\#2, active power control is translated into current control by (2). After this, $i_{d-b a t}^{*}$ is assigned to the current control loop as the reference value.

\section{Power Exchange Estimation Based on Power Forecasting}

Another important point of the proposed framework is that the micro-grid is controlled to exchange predefined amount of power $P_{e x}$ with the utility grid through regulating VSC_\#2 in every certain period. The setting value $P_{e x}^{*}$ is estimated based on power forecasting of renewable generation and local loads with consideration of the battery SOC. It is designed in this work that $P_{e x}^{*}$ is updated every 15 minutes and broadcasted to the utility grid operators at the same time. In this case, the microgrid becomes a predictable $P Q$ node for the utility grid. Furthermore, the power system can actively decide how many, when and which of these micro-grids are permitted to access or quit with more flexibility and confidence. This will greatly benefit the dispatching algorithm of the power system.

Forecasting of solar power can be divided into three categories according to the time horizons, very-short term, short-term and long-term. Very-short term forecasting generally refers to that lasts from seconds to 30 minutes and is mainly used in real-time power regulations [20]-[22]. In the proposed micro-grid framework, power forecasting is part of the operation strategy and the time scale is set to 15 minutes, which is also the updating interval of $P_{e x}^{*}$.

The most favored theories in power forecasting include artificial neural networks (ANN), support vector machine (SVM), autoregressive integrated moving average (ARIMA) etc. [23]-[24]. In solar stations, historical data is generally recorded as time series with different intervals. ARIMA performs effectively in accuracy if these data has a linear or higher order inherent pattern, especially in very-short term forecasting. Actually, solar power is sensitive the sunlight intensity and ambient temperature, and its output always contains stochastic variations due to the weather conditions [25][26].

This work applies a curve fitting approach to predict the solar power generation in the next 15 minutes. The historical data of the latest periods are denoted as:

$$
\left(t_{i}, P_{i}\right), i=0,1,2 \ldots n-1
$$


where $t_{i}$ is the discrete time moments and $P_{i}$ is the corresponding power generation. Based on these data, we attempt to find an approximate continuous univariate function $f(t)$ to describe their relation and further to forecast the future power outputs. The basic form of this function is expressed as (10).

$$
f(t)=\sum_{j=0}^{m-1} \alpha_{j} \varphi_{j}(t)=\alpha_{0} \varphi_{0}(t)+\alpha_{1} \varphi_{1}(t)+\cdots+\alpha_{m-1} \varphi_{m-1}(t)
$$

where $\varphi(t)$ belongs to a specific linear-independence function space $\Phi$, and $\alpha$ is the coefficient of each sub-function. Generally, $\Phi$ is a polynomial function space $\Phi=\left\{1, t_{1}, t_{2} \cdots t_{m-1}\right\}$ and (10) is rewritten as (11). The residual error at each data point is calculated by (12).

$$
\begin{gathered}
f(t)=\sum_{j=0}^{m-1} \alpha_{j} t^{j} \\
e_{i}=P_{i}-f\left(t_{i}\right), \quad i=0,1,2 \cdots n-1
\end{gathered}
$$

To evaluate the accuracy of this fitting function $f(t)$, the least 2-Norm criteria is employed as in (13):

$$
\|e\|_{2}^{2}=\sum_{i=0}^{n-1} e_{i}^{2}=\sum_{i=0}^{n-1}\left(P_{i}-f\left(t_{i}\right)\right)^{2}=\sum_{i=0}^{n-1}\left(P_{i}-\sum_{j=0}^{m-1} \alpha_{j} t_{i}^{j}\right)^{2}=\min
$$

$\|e\|_{2}^{2}$ is a multivariate function of variables $\left(\alpha_{0}, \alpha_{1}, \alpha_{2} \cdots \alpha_{\mathrm{m}-1}\right)$, which are the coefficients of $f(t)$. Final selected results of $\left(\alpha_{0}, \alpha_{1}, \alpha_{2} \cdots \alpha_{\mathrm{m}-1}\right)$ should guarantee that $\|e\|_{2}^{2}$ gets its minimum value. This is a multiextremum optimization problem, and the partial derivatives of $\|e\|_{2}^{2}$ for each variable should be zero. The partial derivatives are calculated by (14) and rewritten as (15).

$$
\begin{gathered}
\frac{\partial\|e\|_{2}^{2}}{\partial \alpha_{j}}=-2 \sum_{i=0}^{n-1}\left(P_{i}-\sum_{k=0}^{m-1} \alpha_{k} t_{i}^{k}\right) \times t_{i}^{j}=0 \\
\sum_{i=0}^{n-1} P_{i} t_{i}^{j}=\sum_{i=0}^{n-1}\left(\sum_{k=0}^{m-1} \alpha_{k} t_{i}^{k+j}\right), \quad j=0,1,2 \cdots m-1
\end{gathered}
$$

Expression (15) is a series of equations and separated into (16). The coefficient matrix of (16) is symmetric and positive definite, and unique values of $\left(\alpha_{0}, \alpha_{1}, \alpha_{2} \cdots \alpha_{\mathrm{m}-1}\right)$ can be derived consequently. Then the curve fitting function $f(t)$ is finally obtained using the least square method. This function will be utilized to predict the future solar power output.

$$
\left[\begin{array}{cccc}
n & \sum_{i=0}^{n-1} t_{i} & \cdots & \sum_{i=0}^{n-1} t_{i}^{m-1} \\
\sum_{i=0}^{n-1} t_{i} & \sum_{i=0}^{n-1} t_{i}^{2} & \cdots & \sum_{i=0}^{n-1} t_{i}^{m} \\
\vdots & \vdots & & \vdots \\
\sum_{i=0}^{n-1} t_{i}^{m-1} & \sum_{i=0}^{n-1} t_{i}^{m} & \cdots & \sum_{i=0}^{n-1} t_{i}^{2(m-1)}
\end{array}\right]\left[\begin{array}{c}
\alpha_{0} \\
\alpha_{1} \\
\vdots \\
\alpha_{m-1}
\end{array}\right]=\left[\begin{array}{c}
\sum_{i=0}^{n-1} P_{i} \\
\sum_{i=0}^{n-1} P_{i} t_{i}^{1} \\
\vdots \\
\sum_{i=0}^{n-1} P_{i} t_{i}^{m-1}
\end{array}\right]
$$

In this work, historical data in the past 30 minutes are chosen for curve fitting. With a time interval of 5 minutes, 7 data points $(n=6)$ are collected totally. The sketch map of the solar power forecasting process is displayed in Fig.8. With the recorded 7 discrete data from $\left(t_{0}, P_{0}\right)$ to $\left(t_{6}, P_{6}\right)$, curve fitting function $f(t)$ is derived. Then in the future 15 minutes, output power at every certain moment can be predicted as $P<P V$. The average value of $P_{<P \vee>}$ is calculated by (17) and used for $P_{e x}^{*}$ estimation.

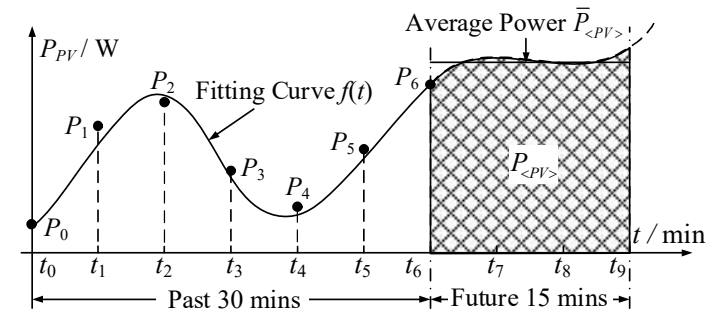

Figure. 8. Sketch map of the solar power forecasting. 


$$
\bar{P}_{<P V>}=\frac{1}{t_{9}-t_{6}} \int_{t_{6}}^{t_{9}} P_{<P V>} d t
$$

As to the local load forecasting, the same curve fitting approach is applied in this work. In the future 15 minutes, load consumption can be predicted as $P<>$. The average value of $P_{<\triangleright>}$ is calculated by (18) and used for $P_{e x}^{*}$ estimation.

$$
\bar{P}_{<l>}=\frac{1}{t_{9}-t_{6}} \int_{t_{6}}^{t_{9}} P_{<l>}(t) d t
$$

With the predicted $\bar{P}_{\langle P V\rangle}$ and $\bar{P}_{\langle l>}$, the predicted average surplus power $\bar{P}_{<o p\rangle}$ is calculated by (19). As mentioned previously, the diesel genset has a minimum power limit $P_{D E}$, which is equipped for the critical load. This component should be included here in (19).

$$
\bar{P}_{<o p>}=\bar{P}_{<P V>}-\left(\bar{P}_{<l>}-P_{D E}\right)
$$

Before assigning $\bar{P}_{<o p>}$ to VSC_\#2 as the set-point of $P_{e x}$, another power component $P_{S O C}$ for the battery balance is designed and added. In Section II, the first two restrictive rules of (5) are considered in the battery control. To avoid over charge/discharge, the battery SOC will be regulated indirectly through updating the set-point of $P_{e x}$. Then $P_{e x}^{*}$ is finally derived by (20) and the designed Psoc curve is displayed in Fig.9.

$$
P_{e x}^{*}=\bar{P}_{<o p>}+P_{S O C}=\bar{P}_{<P V>}+P_{D E}-\bar{P}_{<l>}+P_{S O C}
$$

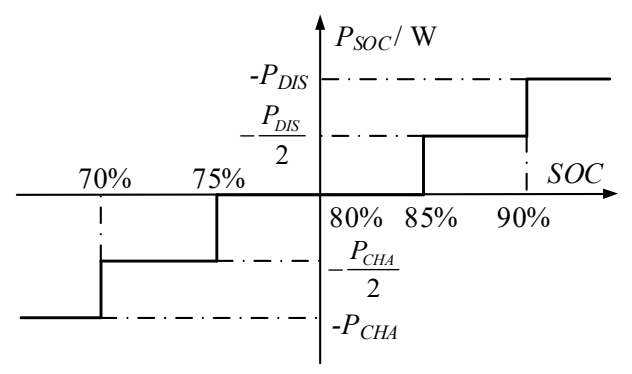

Figure. 9. Designed PSOC curve for the battery balance.

To retain sufficient capability of the battery for power smoothing in every period (15 minutes), the baseline SOC of the battery is defined as $80 \%$. At the beginning of every updating period, the realtime SOC of the battery is estimated for PSOC calculation. As displayed in Fig.9 as well as (21), a tolerable range is designed as [75\%, 85\%], in which $P$ soc equals to zero. Whereas if SOC goes beyond either limit, a corresponding constant value will be set to $P_{S O C}$. For instance, if SOC $>85 \%$ and locates within the range of $[85 \%, 90 \%]$, a constant positive value $-P_{D I S} / 2$ is then added into $P_{e x}^{*}$ which indicates that more power will be transmitted from the micro-grid to the utility grid and the battery is supposed to be discharged in the next 15 minutes. If the SOC $>90 \%$, then a larger value $-P_{D I S}$ will be generated. $P_{C H A}$ and $P_{D I S}$ are the charging and discharging limits of the battery storage, respectively.

$$
P_{S O C}=\left\{\begin{array}{cc}
-P_{D I S} & S O C \geq 90 \% \\
-\frac{P_{D I S}}{2} & 85 \% \leq S O C<90 \% \\
0 & 75 \% \leq S O C<85 \% \\
-\frac{P_{C H A}}{2} & 70 \% \leq S O C<75 \% \\
-P_{C H A} & S O C<70 \%
\end{array}\right.
$$

\section{Results and Verifications}

A typical AC micro-grid model with the same structure in Fig.1 is built in this work, and the capacity of each unit is listed in Table I. The line impedances $Z_{1} \sim Z_{4}$ has a resistance $0.092 \Omega$ and an inductance $125 \mu \mathrm{H}$ to simulate a $50 \mathrm{~m}$ transmission line (line diameter is about $15 \mathrm{~mm}$ ). The nominal AC BUS voltage is $380 \mathrm{~V} / 50 \mathrm{~Hz}$ (line-to-line). 
Table 1. Capacity allocation of each unit

\begin{tabular}{ll}
\hline \multicolumn{1}{c}{ Units } & Capacity \\
\hline Diesel genset & $100 \mathrm{kVA}$ \\
Linear load & $28 \mathrm{~kW}$ \\
Converter load & $95 \mathrm{kWp}$ \\
Solar power & $115 \mathrm{kWp}$ \\
Battery & $700 \mathrm{~V} / 30 \mathrm{Ah}$ \\
\hline
\end{tabular}

In simulation, the diesel genset is started first together with the critical load, and then is the battery storage, the solar array and the converter load in sequence. The AC/DC/AC converter is the last to be activated. Performance waveforms are presented below to verify the proposed framework and operation strategy.

The varying solar power $P_{P V}$ and residential loads $P_{l}$ are displayed in Fig.10 (a) and (b). Based on the historical data in the latest 30 minutes, the solar power $P<P V>$ and load $P<l>$ in the next 15 minutes can be predicted using the curve fitting forecasting method. Then the average value $\bar{P}_{<P V\rangle}$ and $\bar{P}_{<l>}$ are calculated for $P_{e x}^{*}$ estimation. This forecasting and calculation process rolls forward every 15 minutes interval. $\bar{P}_{<P V\rangle}$ and $\bar{P}_{<l>}$ are also updated every 15 minutes.
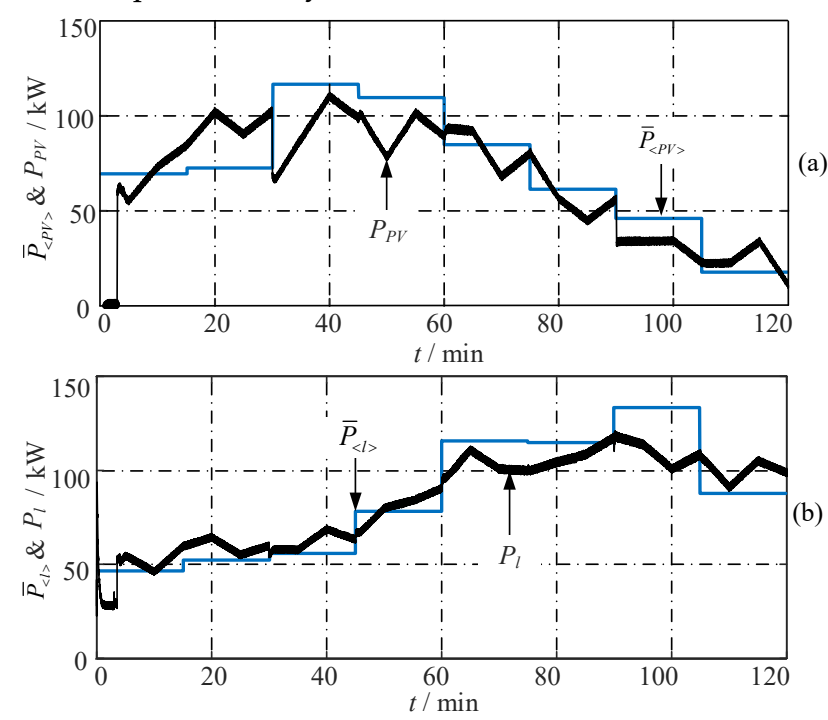

Figure. 10. Varying solar power and residential loads and their predicted average values; (a) solar power $P_{P V}$ and $\bar{P}_{<P V\rangle} ;\left(\right.$ b) residential loads $P_{l}$ and $\bar{P}_{\langle l\rangle}$.

The DC/AC/DC converter is regulated to exchange predefined amount of power $P_{e x}$ with the utility grid in every 15 minutes. The reference set-point $P_{e x}^{*}$ for VSC_\#2 is calculated by (20), where $P_{D E}=28 \mathrm{~kW}$ and $P_{S O C}$ is generated by (21). The power delivery of the micro-grid $P_{e x}$ is displayed in Fig. 11 (a), where the power exchange between the two utilities is predefined and broadcasted every 15 minutes. From the view of the utility grid, every micro-grid becomes a $P Q$ node, which will benefit the dispatching algorithm of the power system. The DC link of the DC/AC/DC converter is equipped with a $1.5 \mathrm{mF}$ capacitor and the rated DC link voltage is $750 \mathrm{~V}$. As displayed in Fig.11 (b), the DC link voltage is maintained $750 \mathrm{~V}$ during operation with only some dynamics.

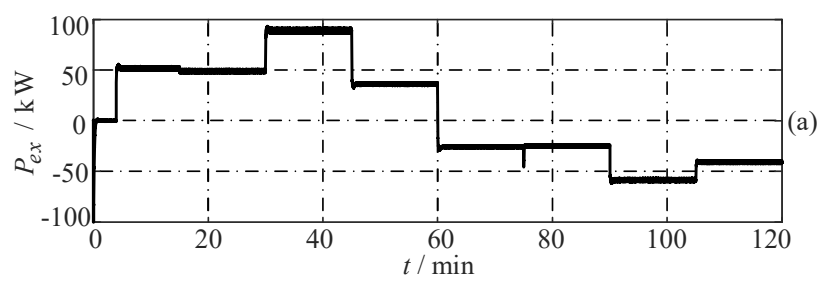




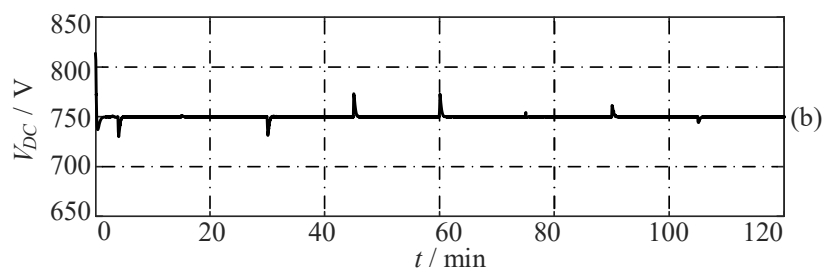

Figure. 11. Performances of the DC/AC/DC converter; (a) power exchange between the micro-grid and the utility grid $P_{e x}$; (b) DC link voltage $V_{D C}$.

The volatile power from renewable sources and local loads are constrained and superseded within the micro-grid circumstances. The battery is to take this responsibility. With the proposed SOC balancing approach, the performances of the battery storage unit with and without balancing are displayed below in Fig.12.
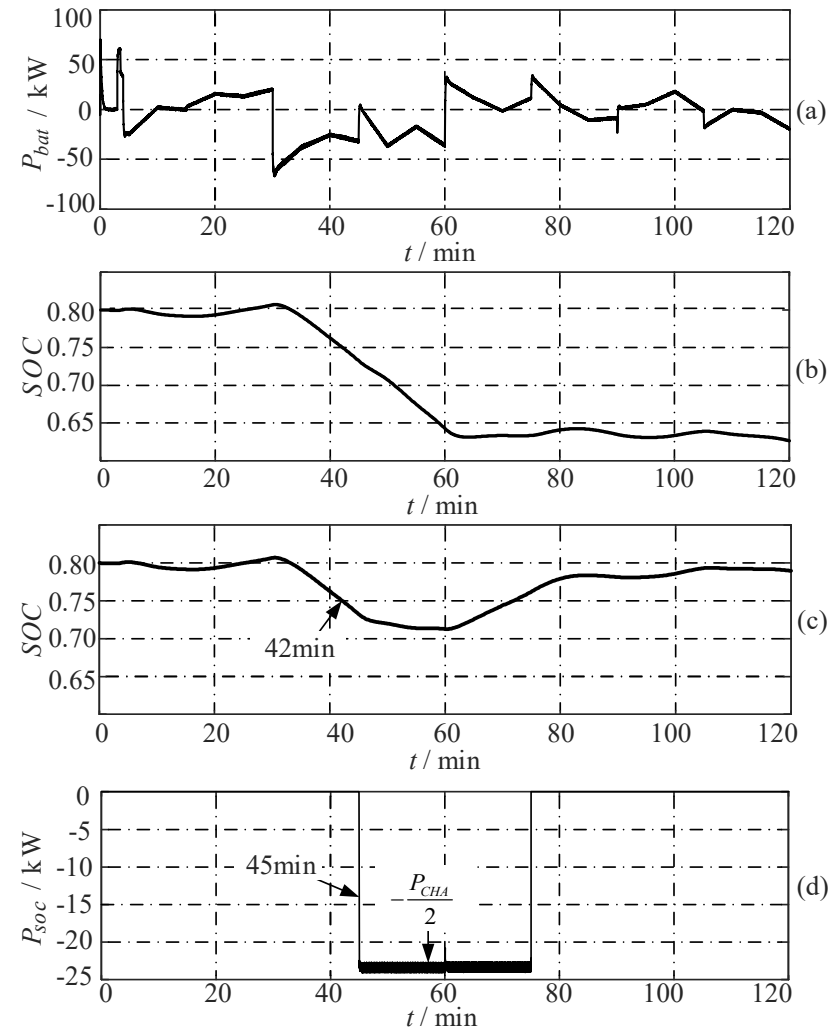

Figure. 12. Performances of the battery storage unit; (a) suppressed power of the battery storage unit $P_{\text {bat }}$; (b) SOC of battery without balancing; (c) SOC of battery with balancing; (d) PSOC for battery balancing.

In Fig.12 (a), all the varying power components that calculated by (4) are compensated by the battery. Without balancing, its SOC increases and decreases irregularly according to the amount of the net power. In this test scenario, it drops to a lower level of $65 \%$ as displayed in Fig.12 (b). Whereas with the designed balancing technique, the SOC of battery can be restored automatically during operation. In Fig.12 (b), SOC drops to the lower limit of the tolerance range $75 \%$ at $42 \mathrm{~min}$, then at the beginning of the next updating interval (at $45 \mathrm{~min}$ ), a corresponding $P_{S O C}=-P_{C H A} / 2$, which equals to $-24 \mathrm{~kW}$ is generated by (21) as displayed in Fig.12 (d). With this adjustment, the SOC of the battery recovers to the tolerance range automatically as displayed in Fig.12 (c). This indicates that the all the varying power components are constrained within the micro-grid circumstances without leaking to the utility grid.

In the operation strategy, the AC BUS of the micro-grid is dominated by diesel genset all along in both operation modes and fully-autonomous operation is finally achieved. A minimum power output limit $P_{D E}=28 \mathrm{~kW}$ is preset for the diesel genset to avoid low load-rate running. Operation 
results are presented in Fig13 (a) and (b). The diesel genset keeps providing 28kW power even with sufficient solar power generations. The system frequency is maintains at its rated value $50 \mathrm{~Hz}$ steadily in the whole test scenario.
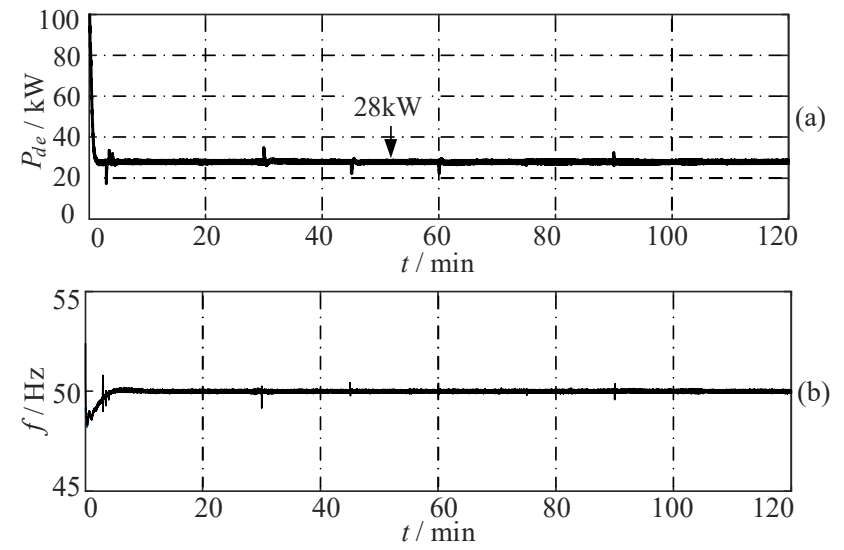

Figure. 13. System performance of the micro-grid; (a) output power of the diesel genset during operation; (b) system frequency $f$ of the micro-grid.

\section{Conclusions}

Conventional grid-connected micro-grid operates non-autonomously and relies on the utility grid for AC BUS supporting and power balancing, which inherits some great challenges in system operation and practical application. This work has proposed a fully-autonomous framework, which applies an AC/DC/AC converter as the interface unit. This unit enables the micro-grid to behave as a fully independent entity in both operation modes as a self-governed entity. In the operation strategy, the $\mathrm{AC} / \mathrm{DC} / \mathrm{AC}$ converter is regulated to exchange predefined amount of power with the utility grid. The power amount is estimated by a curve fitting based power forecasting approach, and updated and broadcasted every 15 minutes. All the varying components are constrained and suppressed within the micro-grid mainly by the battery storage with a SOC balancing approach. The proposed framework and operation strategy can improve the system reliability and also benefit the dispatching algorithm of the power system, which makes a step forward to the high penetration depth application of renewables sources into the utility grid. Simulation results are finally provided for a verification.

Author Contributions: Conceptualization, Baoquan Liu and Yixin Zhu; methodology, Baoquan Liu; software, Yan Liu; validation, Yan Liu; investigation, Baoquan Liu; data curation, Yan Liu; writing-original draft preparation, Baoquan Liu; writing - review and editing, Yong Shi; supervision, Yong Shi

Funding: This research received no external funding.

Conflicts of Interest: The authors declare no conflict of interest. The funders had no role in the design of the study; in the collection, analyses, or interpretation of data; in the writing of the manuscript, or in the decision to publish the results.

\section{References}

1. Robert H. Lasseter and Paolo Paigi, “Microgrid: A conceptual solution,” in Proc. 2004 IEEE Power Electronics Specialists Conf., pp. 4285-4290.

2. L.A. de Souza Ribeiro, O.R. Saavedra, S.L. de Lima and J. Gomes de Matos, "Isolated Micro-Grids with Renewable Hybrid Generation: The Case of Lençóis Island," IEEE Trans. Sustainable Energy, vol. 2, pp. 111, Jan. 2011.

3. H. Shi, F. Zhuo, H. Yi, F. Wang, D. Zhang and Z. Geng, "A Novel Real-Time Voltage and Frequency Compensation Strategy for Photovoltaic-Based Microgrid," IEEE Transactions on Industrial Electronics, vol. 62, no. 6, pp. 3545-3556, June 2015.

4. Y. Zhu, F. Zhuo, F. Wang, B. Liu and Y. Zhao, "A Wireless Load Sharing Strategy for Islanded Microgrid Based on Feeder Current Sensing," in IEEE Transactions on Power Electronics, vol. 30, no. 12, pp. 6706-6719, Dec. 2015. 
5. Dong Chen and Lie $\mathrm{Xu}$, “Autonomous DC voltage control of a DC Microgrid with multiple slack terminals," IEEE Trans. Power Systems, vol. 27, pp. 1897-1905, Nov. 2012.

6. X. Liu, M. Shahidehpour, Z. Li, X. Liu, Y. Cao and Z. Bie, "Microgrids for Enhancing the Power Grid Resilience in Extreme Conditions," IEEE Transactions on Smart Grid, vol. 8, no. 2, pp. 589-597, March 2017.

7. C. Dou, N. Li, D. Yue and T. Liu, "Hierarchical hybrid control strategy for micro-grid switching stabilization during operating mode conversion," IET Generation, Transmission \& Distribution, vol. 10, no. 12, pp. 28802890, 2016.

8. S. S. Thale and V. Agarwal, "Controller Area Network Assisted Grid Synchronization of a Microgrid With Renewable Energy Sources and Storage," IEEE Transactions on Smart Grid, vol. 7, no. 3, pp. 1442-1452, May 2016.

9. S. M. Malik, X. Ai, Y. Sun, C. Zhengqi and Z. Shupeng, "Voltage and frequency control strategies of hybrid AC/DC microgrid: a review," in IET Generation, Transmission \& Distribution, vol. 11, no. 2, pp. 303-313, 2017.

10. F. Nejabatkhah and Y. W. Li, "Overview of Power Management Strategies of Hybrid AC/DC Microgrid," IEEE Transactions on Power Electronics, vol. 30, no. 12, pp. 7072-7089, Dec. 2015.

11. A. Micallef, M. Apap, C. Spiteri-Staines and J. M. Guerrero, "Single-Phase Microgrid With Seamless Transition Capabilities Between Modes of Operation," IEEE Transactions on Smart Grid, vol. 6, no. 6, pp. 2736-2745, Nov. 2015.

12. M. N. Arafat, A. Elrayyah and Y. Sozer, "An Effective Smooth Transition Control Strategy Using DroopBased Synchronization for Parallel Inverters," IEEE Transactions on Industry Applications, vol. 51, no. 3, pp. 2443-2454, May-June 2015.

13. Y. A. R. I. Mohamed and A. A. Radwan, "Hierarchical Control System for Robust Microgrid Operation and Seamless Mode Transfer in Active Distribution Systems," IEEE Transactions on Smart Grid, vol. 2, no. 2, pp. 352-362, June 2011.

14. B. Liu, F. Zhuo, Y. Zhu and H. Yi, "System Operation and Energy Management of a Renewable EnergyBased DC Micro-Grid for High Penetration Depth Application," IEEE Transactions on Smart Grid, vol. 6, no. 3, pp. 1147-1155, May 2015.

15. S. Pelland, D. Turcotte, G. Colgate and A. Swingler, "Nemiah Valley Photovoltaic-Diesel Mini-Grid: System Performance and Fuel Saving Based on One Year of Monitored Data," IEEE Transactions on Sustainable Energy, vol. 3, no. 1, pp. 167-175, Jan. 2012.

16. C. Ahumada, R. Cárdenas, D. Sáez and J. M. Guerrero, "Secondary Control Strategies for Frequency Restoration in Islanded Microgrids With Consideration of Communication Delays," IEEE Transactions on Smart Grid, vol. 7, no. 3, pp. 1430-1441, May 2016.

17. D. I. Brandao, T. Caldognetto, F. P. Marafão, M. G. Simões, J. A. Pomilio and P. Tenti, "Centralized Control of Distributed Single-Phase Inverters Arbitrarily Connected to Three-Phase Four-Wire Microgrids," IEEE Transactions on Smart Grid, vol. 8, no. 1, pp. 437-446, Jan. 2017.

18. H. Han, X. Hou, J. Yang, J. Wu, M. Su and J. M. Guerrero, "Review of Power Sharing Control Strategies for Islanding Operation of AC Microgrids," IEEE Transactions on Smart Grid, vol. 7, no. 1, pp. 200-215, Jan. 2016.

19. K. V. Vidyanandan and N. Senroy, "Frequency regulation in a wind-diesel powered microgrid using flywheels and fuel cells," IET Generation, Transmission E Distribution, vol. 10, no. 3, pp. 780-788, 2182016.

20. I. P. Panapakidis and G. C. Christoforidis, "A hybrid ANN/GA/ANFIS model for very short-term PV power forecasting," 2017 11th IEEE International Conference on Compatibility, Power Electronics and Power Engineering (CPE-POWERENG), Cadiz, 2017, pp. 412-417.

21. J. Tian, Z. Liu, J. Shu, J. Liu and J. Tang, "Base on the ultra-short term power prediction and feed-forward control of energy management for microgrid system applied in industrial park," IET Generation, Transmission \& Distribution, vol. 10, no. 9, pp. 2259-2266, 2016.

22. A. Bracale, G. Carpinelli and P. De Falco, "A Probabilistic Competitive Ensemble Method for Short-Term Photovoltaic Power Forecasting," IEEE Transactions on Sustainable Energy, vol. 8, no. 2, pp. 551-560, April 2017.

23. J. Shi, W. J. Lee, Y. Liu, Y. Yang and P. Wang, "Forecasting Power Output of Photovoltaic Systems Based on Weather Classification and Support Vector Machines," IEEE Transactions on Industry Applications, vol. 48, no. 3, pp. 1064-1069, May-June 2012.

24. M. G. De Giorgi, P. M. Congedo and M. Malvoni, "Photovoltaic power forecasting using statistical methods: impact of weather data," in IET Science, Measurement $\mathcal{E}$ Technology, vol. 8, no. 3, pp. 90-97, May 2014. 
25. D. van der Meer, G. R. Chandra Mouli, G. Morales-Espana, L. Ramirez Elizondo and P. Bauer, "Energy Management System with PV Power Forecast to Optimally Charge EVs at the Workplace," IEEE Transactions on Industrial Informatics (early access paper).

26. C. E. Borges, Y. K. Penya and I. Fernández, "Evaluating Combined Load Forecasting in Large Power Systems and Smart Grids," in IEEE Transactions on Industrial Informatics, vol. 9, no. 3, pp. 1570-1577, Aug. 2013. 